\title{
Caffeic acid phenethyl ester promotes haematopoietic stem/progenitor cell homing and engraftment
}

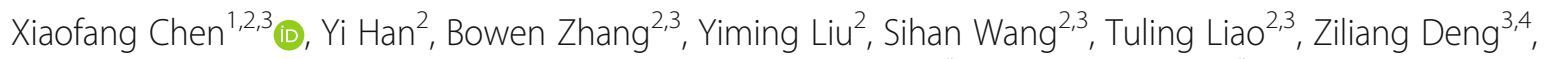
Zeng Fan ${ }^{2,3}$, Jing Zhang ${ }^{2,3}$, Lijuan He ${ }^{2,3}$, Wen Yue ${ }^{2,3}$, Yanhua Li $i^{2,3^{*}}$ and Xuetao Pei ${ }^{1,2,3^{*}}$

\begin{abstract}
Background: Several studies have suggested that caffeic acid phenethyl ester (CAPE) can induce the expression of hypoxia inducible factor-1a (HIF-1a) protein. We determined whether CAPE has a novel function in improving the homing and engraftment of haematopoietic stem/progenitor cells (HSPCS) by regulating HIF-1a gene expression in the bone marrow (BM) niche.

Methods: For survival experiments, lethally irradiated C57BL/6 mice were injected with a low number of BM mononuclear cells (MNCs) and CAPE according to the indicated schedule. Homing efficiency analysis was conducted using flow cytometry and colony-forming unit (CFU) assays. The influence of intraperitoneal injection of CAPE on short-term and long-term engraftment of HSPCs was evaluated using competitive and non-competitive mouse transplantation models. To investigate the mechanism by which CAPE enhanced HSPC homing, we performed these experiments including Q-PCR, western blot, immunohistochemistry and CFU assays after in-vivo HIF-1a activity blockade.

Results: CAPE injection significantly increased the survival rate of recipient mice after lethal irradiation and transplantation of a low number of BM MNCs. Using HSPC homing assays, we found that CAPE notably increased donor HSPC homing to recipient BM. The subsequent short-term and long-term engraftment of transplanted HSPCs was also improved by the optimal schedule of CAPE administration. Mechanistically, we found that CAPE upregulated the expression of HIF-1a, vascular endothelial growth factor-A (VEGF-A) and stromal cell-derived factor 1a (SDF-1a). The HIF-1a inhibitor PX-478 blocked CAPE-enhanced HSPC homing, which supported the idea that HIF-1a is a key target of CAPE.
\end{abstract}

Conclusions: Our results showed that CAPE administration facilitated HSPC homing and engraftment, and this effect was primarily dependent on HIF-1a activation and upregulation of SDF-1a and VEGF-A expression in the BM niche.

Keywords: Caffeic acid phenethyl ester, Haematopoietic stem/progenitor cells, Homing, Engraftment

\footnotetext{
*Correspondence: shirlylyh@126.com; peixt@nic.bmi.ac.cn

${ }^{2}$ Stem Cell and Regenerative Medicine Lab, Beijing Institute of Transfusion

Medicine, No. 27 Taiping Road, Haidian District, Beijing 100850, China

${ }^{1}$ School of Laboratory Medicine and Biotechnology, Southern Medical

University, No. 1838 Guangzhou Avenue North, Baiyun District, Guangzhou

510515, China

Full list of author information is available at the end of the article
} 


\section{Background}

Haematopoietic stem cell transplantation (HSCT) has been applied for treating malignant and nonmalignant haematologic diseases in the clinic for over 50 years [1, 2]. However, the curative rate of this treatment, especially for cord blood (CB) HSCT, must be improved because of the low homing efficiency of haematopoietic stem and progenitor cells (HSPCs), delayed engraftment and the occurrence of graft versus host diseases. Efficient homing and engraftment of HSPCs are critical for haematopoietic repopulation in patients after transplantation, and these processes depend on several important steps. First, infused HSPCs find their way to bone marrow (BM) microvessels via rolling and firm adhesion to endothelial cells. Subsequently, the HSPCs migrate across the BM endothelial cells (BMECs) and extracellular matrix barrier and lodge in the BM niche. Complicated molecular interactions are involved in the homing process, including selection with its ligand, chemotactic SDF-1/CXCR4 axis and adherent VCAM-1/VLA4 axis and so on [3-7]. Finally, the transplanted HSPCs undergo proliferation and multilineage differentiation in their BM home and reconstitute the haematopoietic and immune systems in the recipients.

Improving HSPC homing to the BM is important for facilitating subsequent engraftment and haematopoietic repopulation, particularly when the number of infused HSPCs is limited [8]. Based on the understanding of the mechanism of HSPC homing, several approaches have been reported to promote the seeding of HSPCs to the $\mathrm{BM}$ and enhance engraftment. Ex-vivo manipulation of HSPCs through incubation with prostaglandin E2 (PGE2), BMP4 or fucosyltransferase-VI can accelerate HSPC homing and engraftment in the BM [9-12]. Accumulated evidence shows that the chemoattractant factor SDF- $1 \alpha$ plays a crucial role in HSPC homing and subsequent engraftment [13-19]. Although several agents regulate $\mathrm{HSPC}$ seeding to the $\mathrm{BM}$ and enhance their engraftment, encouraging more HSPCs to find their way to the $\mathrm{BM}$ is still a big challenge.

Caffeic acid phenethyl ester (CAPE) is a propolis extract obtained from honeybee hives and an ester analogue of caffeic acid. Increasing evidence indicates that CAPE has multiple beneficial properties, such as antibacterial, anti-viral, anti-inflammatory and anticancer properties [20-25]. Previous studies have suggested that CAPE can induce the expression of hypoxia inducible factor- $1 \alpha$ (HIF-1 $\alpha)$ protein, which occurred by inhibition of HIF prolyl hydroxylase, the key enzyme for von Hippel-Lindau-dependent HIF-1 $\alpha$ degradation, and activate the expression of HIF- $1 \alpha$ target genes, such as vascular endothelial growth factor (VEGF) and haeme oxygenase-1 (HO-1), which play protective roles in ischemia/reperfusion injury [26-28]. In our previous study, we found that CAPE upregulated the expression of HIF- $1 \alpha$ and stem cell factor (SCF) to promote the expansion of HSPCs in vitro [29]. Since HIF- $1 \alpha$, an upstream transcriptional factor, regulates the expression of SDF- $1 \alpha$ and because SDF- $1 \alpha$ is a critical chemotactic factor for HSPC migration, we speculate that CAPE may enhance HSPC homing to the BM.

Herein, we show that CAPE administration to irradiated recipients promotes the recruitment and homing of HSPCs to the BM niche in a BM transplantation model. The subsequent enhanced short-term and long-term engraftment of transplanted HSPCs was also improved by the optimal schedule of CAPE administration. This occurs primarily via HIF- $1 \alpha$ activation and by affecting the expression of SDF- $1 \alpha$ and VEGF in the BM niche. Thus, we present a novel role for CAPE: improving the homing and engraftment of HSPCs.

\section{Methods \\ Mice}

C57BL/6-CD45.2 mice (CD45.2 mice, 6-8 weeks old, male, 20-24 g) were purchased from Beijing Vital River Laboratory Animal Technology Company, Ltd. C57BL/6-CD45.1 mice (CD45.1 mice, 6-8 weeks old, male, 20-24 g) were purchased from the Institute of Haematology, Chinese Academy of Medical Sciences (Tianjin, China). All animal experiments were reviewed and approved by the Animal Center Committee of the Academy of Military Medical Sciences (Beijing, China). After lethal irradiation, mice were fed with distilled water containing gentamicin.

\section{Radiation and treatment}

Mice were lethally irradiated ( $950 \mathrm{cGy}, 80-120 \mathrm{cGy} / \mathrm{min}$ ) using a ${ }^{60} \mathrm{Co}$ irradiator. CAPE (Sigma) concentrated solution was prepared in saline containing 5\% dimethyl sulphoxide (DMSO; Sigma), 20\% propanediol and 0.2\% Tween 80. Mice were injected intraperitoneally with CAPE daily at the indicated doses for 3 days-that is, day -1 ( $24 \mathrm{~h}$ before irradiation), day 0 ( $0 \mathrm{~h}$ after irradiation) and day +1 ( $24 \mathrm{~h}$ after irradiation) - while the control mice were injected intraperitoneally with the equal volume of vehicle used for CAPE dissolution. The HIF- $1 \alpha$ inhibitor PX-478 (Xcess Biosciences) was dissolved in DMSO at a concentration of $20 \mu \mathrm{g} / \mu \mathrm{l}$. Mice were injected intraperitoneally with $5 \mathrm{mg} / \mathrm{kg}$ PX-478 in PBS just after daily CAPE injections.

\section{Transplantation experiments}

For survival experiments, lethally irradiated mice were injected with $2.5 \times 10^{5}$ bone marrow mononuclear cells (BM MNCs) and CAPE according to the indicated schedule. For homing experiments, lethally irradiated CD45.2 mice were injected intraperitoneally with vehicle 
or $3.0 \mathrm{mg} / \mathrm{kg}$ CAPE for 3 days. Twenty hours after irradiation, these recipient mice were injected with $2 \times 10^{7}$ BM MNCs from CD45.1 mice. For CFU-S12 detection, lethally irradiated mice were treated with vehicle or 3.0 $\mathrm{mg} / \mathrm{kg}$ CAPE for 3 days and were injected with $1.5 \times 10^{5}$ BM MNCs 20-24 h after irradiation. For the competitive transplantation experiment, BM MNCs $\left(5 \times 10^{5}\right)$ were harvested from the primary recipient mice $20 \mathrm{~h}$ after bone marrow transplantation (BMT) and the various treatments, and were then mixed with $5 \times 10^{5}$ competitor CD $45.2^{+} \mathrm{BM}$ MNCs and co-transplanted into irradiated CD45.2 mice (secondary recipients).

\section{Immunophenotype analysis using flow cytometry}

BM samples were collected from the femurs and tibias of mice and then lysed with erythrocyte lysing buffer. The remaining cells were first stained with Fixable Viability Stain 510 (FVS510; BD Bioscience). For blood cell lineage detection, the cells were stained with lineage antibodies against CD3e, CD11b, Ter-119, B220, Gr-1 and matched isotype controls (eBioscience). To examine the percentages of LSK $\left(\mathrm{Lin}^{-} \mathrm{Sca}-1^{+} \mathrm{c}-\mathrm{Kit}^{+}\right.$) cells, BM cells were stained with biotin-labelled lineage antibodies and then incubated with streptavidin APC-eFluor ${ }^{\circ} 780$ labelled secondary antibody (eBioscience), along with BV605-labelled anti-mouse Sca-1 (BD Bioscience) and APC-labelled c-Kit (eBioscience). For the detection of engraftment and chimerism, the BM cells were stained with PE or FITC-labelled antibodies against CD45.1 or CD45.2 (eBioscience). All samples were detected by BD FACS Aria (BD Bioscience).

\section{Colony-forming unit assays}

BM MNCs were collected and cultured in MethoCult ${ }^{\mathrm{tm}}$ GF M3434 medium (STEMCELL Technologies). Seven days later, typical colonies, including granulocyte-erythroblast-macrophage-megakaryocyte colony-forming units (CFU-GEMM), erythrocyte burst-forming units (BFU-E), granulocyte-macrophage colony-forming units (CFU$\mathrm{GM}$ ), megakaryocyte colony-forming units (CFU-Meg) and granulocyte colony-forming units (CFU-G), were visually scored based on morphological criteria using a light microscope.

\section{Cytokine array}

BM samples were collected from lethally irradiated C57BL/6 mice $20 \mathrm{~h}$ after $2 \times 10^{7} \mathrm{BM}$ MNC transplantation with vehicle or $3.0 \mathrm{mg} / \mathrm{kg}$ CAPE injection from day -1 to day +1 . The femurs of three mice per group were flushed using $5 \mathrm{ml}$ PBS. The pooled supernatants per group were collected after centrifugation, freezedried and resuspended in $120 \mu \mathrm{l}$ PBS. Cytokine array was performed using the RayBio ${ }^{\circ}$ Mouse Cytokine Antibody Array G-Series 3 (RayBiotech) following the manufacturer's instructions. Data analysis was performed using the analysis tool provided by the kit.

\section{BMEC culture and treatment with CAPE}

BM MNCs were isolated from the femurs and tibias of mice. Primary mouse BMECs were obtained by culturing BM MNCs in $\mathrm{EGM}^{\mathrm{m}}-2-\mathrm{MV}$ BulletKit ${ }^{\mathrm{m}}$ medium (EGM2 medium, CC-3202; Lonza). Non-adherent cells were poured off $72 \mathrm{~h}$ after seeding cells in the medium. The BMECs were identified by immunofluorescence staining using antibodies against CD31 (Proteintech), VECadherin (Abcam), Bs-lectin (Vector Laboratories) and Vwf (Proteintech). Primary BMECs were replated into new six-well plates with EGM2 medium. CAPE, dissolved in DMSO at $10 \mathrm{mg} / \mathrm{ml}$, was added into the culture medium at different concentrations $(0,0.1$ and 1.0 $\mu \mathrm{g} / \mathrm{ml})$. Cells were cultured at $37^{\circ} \mathrm{C}$ in $5 \% \mathrm{CO}_{2}$ for $24 \mathrm{~h}$.

\section{Quantitative RT-PCR}

Total RNA was obtained using TRIzol reagent (Invitrogen), and $1000 \mathrm{pg}$ of RNA was reverse-transcribed into cDNA with ReverTra Ace qPCR RT Master Mix (Toyobo). All gene expression data were normalized by the housekeeping gene, $\beta$-ACTIN. Q-PCR analysis was performed with a Bio-Rad iQ5 system using Thunderbird SYBR qPCR Mix (Toyobo). The primer sequences are presented in Additional file 1: Table S1.

\section{Western blot analysis}

The BMECs were resuspended in buffer containing proteinase inhibitors. The lysates were cleared by centrifugation. The concentration of the total protein per sample was detected using a BCA protein assay kit (Thermo Scientific) and $60 \mu \mathrm{g}$ of protein sample was loaded for western blot analysis using antibodies against mouse $\beta$-ACTIN (1:2000; Cell Signaling Technology), HIF-1 $\alpha$ (1:500; Novus Biologicals), SDF$1 \alpha$ (1:2000; Abcam) and VEGF-A (1:10,000; R\&D Systems).

\section{Immunohistochemistry}

The femurs and tibias of mice were dissected and fixed in $4 \%$ formalin. Bones were dehydrated after decalcification, embedded in paraffin and cut into sections. Immunostaining was performed according to the manufacturer's recommendations (Vector Laboratories). BM sections were stained with primary antibodies, including monoclonal anti-mouse HIF-1 $\alpha$ (1:20; Novus Biologicals), SDF- $1 \alpha$ (1:200; Abcam) or VEGF-A (1:200; R\&D Systems), and biotin-labelled secondary antibodies.

\section{Statistical analysis}

Survival rate data were analysed using a log-rank test (GraphPad Prism 5; GraphPad Software). For 
comparison of two groups, data were analysed with twotailed Student's $t$ tests, and $P<0.05$ was significant. All error bar data represent the mean \pm SD.

\section{Results \\ CAPE increased the survival rate of mice after lethal irradiation and BMT}

To determine whether CAPE treatment had a beneficial effect by regulating HSPC homing, we performed survival rate experiments on mice given CAPE injections. The mice received irradiation at day 0 and BMT at day +1 . To determine an effective CAPE injection schedule, mice were administered $3.0 \mathrm{mg} / \mathrm{kg}$ of CAPE intraperitoneally at different schedules (Fig. 1a). The vehicle control group of mice with lethal irradiation and $2.5 \times 10^{5}$ BM MNC transplantation had the lowest survival rate at 46.7\% (Fig. 1b). The survival rates of the mice treated with CAPE for two doses (from day -1 to day 0 or from day 0 to day +1 ) were $70 \%$ and $80 \%$ separately, both of which were higher than that of the one dose of CAPE group (60\%). We found that daily CAPE treatment for 3 days resulted in a $93.75 \%$ survival rate in mice with lethal irradiation and $2.5 \times 10^{5} \mathrm{BM}$ MNC transplantation (Fig. 1b), which indicated that three injections of CAPE were effective in elevating the survival rates of mice receiving BMT. To further determine the dose-response relationship between CAPE treatment and the improvements in the survival rate of mice receiving BMT, mice were injected with $0,0.3,1.5$ or $3.0 \mathrm{mg} / \mathrm{kg}$ CAPE for 3 consecutive days. The $3.0 \mathrm{mg} / \mathrm{kg}$ CAPE dose was observed to be the optimal dose and resulted in the highest survival rate of the mice with BMT (Fig. 1c). These results indicated that administration of three doses of
CAPE from day -1 to day +1 accelerated haematopoietic repopulation, which might primarily depend on the role of CAPE in regulating HSPC homing.

\section{CAPE promotes HSPC homing to the BM}

To assess whether CAPE promoted HSPC homing to BM niches, lethally irradiated CD45.2 mice were injected intraperitoneally with three doses of vehicle or CAPE, and then BM MNCs isolated from CD45.1 mice were transplanted into the irradiated mice (Fig. 2a). Twenty hours later, BM cells from the recipients were harvested and analysed according to their cell surface marker expression. Using flow cytometry, we found that CAPE treatment led to a $2.4 \pm 0.8$-fold increase in the percentage of CD45. $1^{+} \mathrm{CD} 45.2^{-} \mathrm{Lin}^{-} \mathrm{Sca}-1^{+}$cells in the BM of the recipients $(P=0.0037$; Fig. $2 \mathrm{~b}, \mathrm{c})$. We then used colonyforming unit (CFU) assays to further evaluate the ability of CAPE to regulate HSPC homing to the BM. Compared with the vehicle group, CAPE administration significantly increased the total number of CFUs in the BM of the recipients $20 \mathrm{~h}$ after $\mathrm{BM}$ transplantation (CAPE vs vehicle, $122 \pm 28.7$ vs $74 \pm 12.7$ CFUs per $5 \times 10^{5}$ BM MNCs, $P=0.000057$; Fig. $2 \mathrm{~d}$ ). We also analysed the number of different types of CFUs, including BFU-E, CFU-G/GM/M and CFU-GEMM, in the BM of the recipients. CAPE treatment caused significant increases in the number of CFU-G/GM/M and CFU-GEMM in the BM $(P=0.0143$ and $P=0.0345$; Fig. $2 \mathrm{e})$. The BFU-E number between the two groups showed little increase. We then used a formula to calculate HSPC homing efficiency. The homing efficiency was determined by comparing the number of homed CFUs with the number of CFUs injected [30]. The results showed that the homing

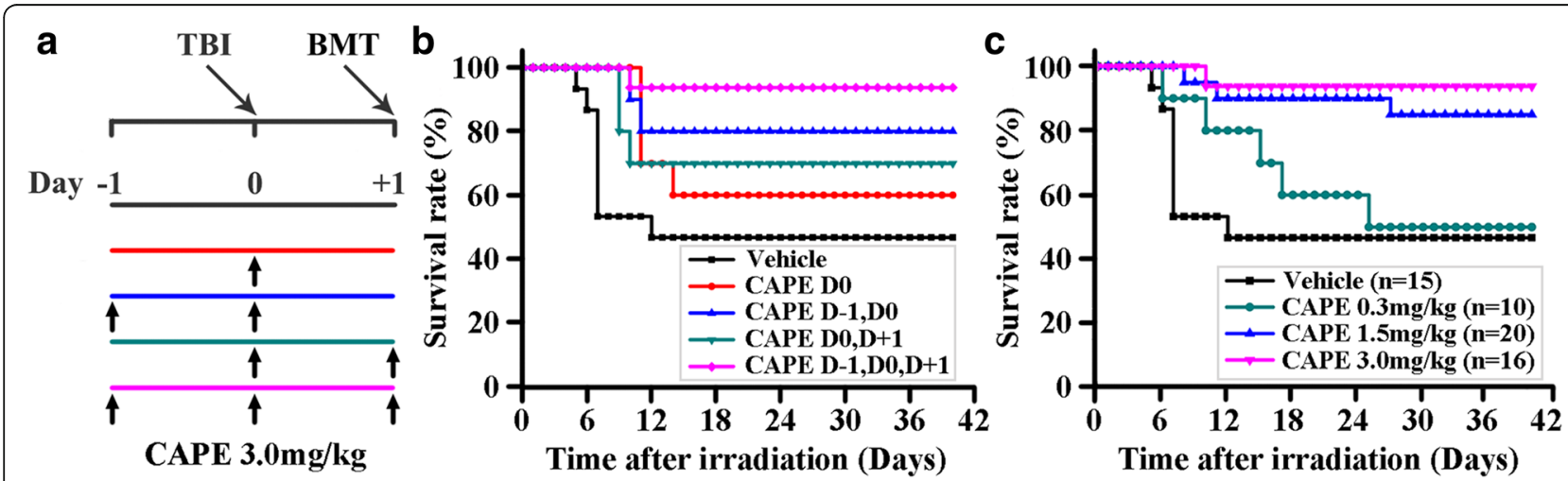

Fig. 1 CAPE increased the survival rate. a BM transplantation and treatment model. All mice were lethally irradiated (950 cGy, $80-120$ cGy/min) on day 0 . Mice were injected intraperitoneally with $3.0 \mathrm{mg} / \mathrm{kg}$ of CAPE at different schedules. Each mouse was transplanted with $2.5 \times 10^{5}$ BM MNCs on day +1 . b Survival rates of the lethally irradiated recipients receiving BMT and CAPE injection with different schedules. All mice were administered vehicle or $3.0 \mathrm{mg} / \mathrm{kg}$ CAPE intraperitoneally. Vehicle: vehicle daily for 3 days $(n=15)$; CAPE D0: vehicle on day -1 and day +1 , CAPE on day 0 ( $n=10)$; CAPE D-1,D0: CAPE on day -1 and day 0 , vehicle on day $+1(n=10)$; CAPE D0,D +1 : vehicle on day -1 , CAPE on day 0 and day $+1(n=10)$; CAPE D-1,D0,D + 1: CAPE daily for 3 days $(n=16)$. c Survival rate of lethally irradiated mice receiving BMT and CAPE injection at different dosages. Lethally irradiated mice were injected with 0 (Vehicle), $0.3,1.5$ or $3.0 \mathrm{mg} / \mathrm{kg}$ CAPE from day -1 to day +1 . TBI total body irradiation, BMT bone marrow transplantation, CAPE caffeic acid phenethyl ester 

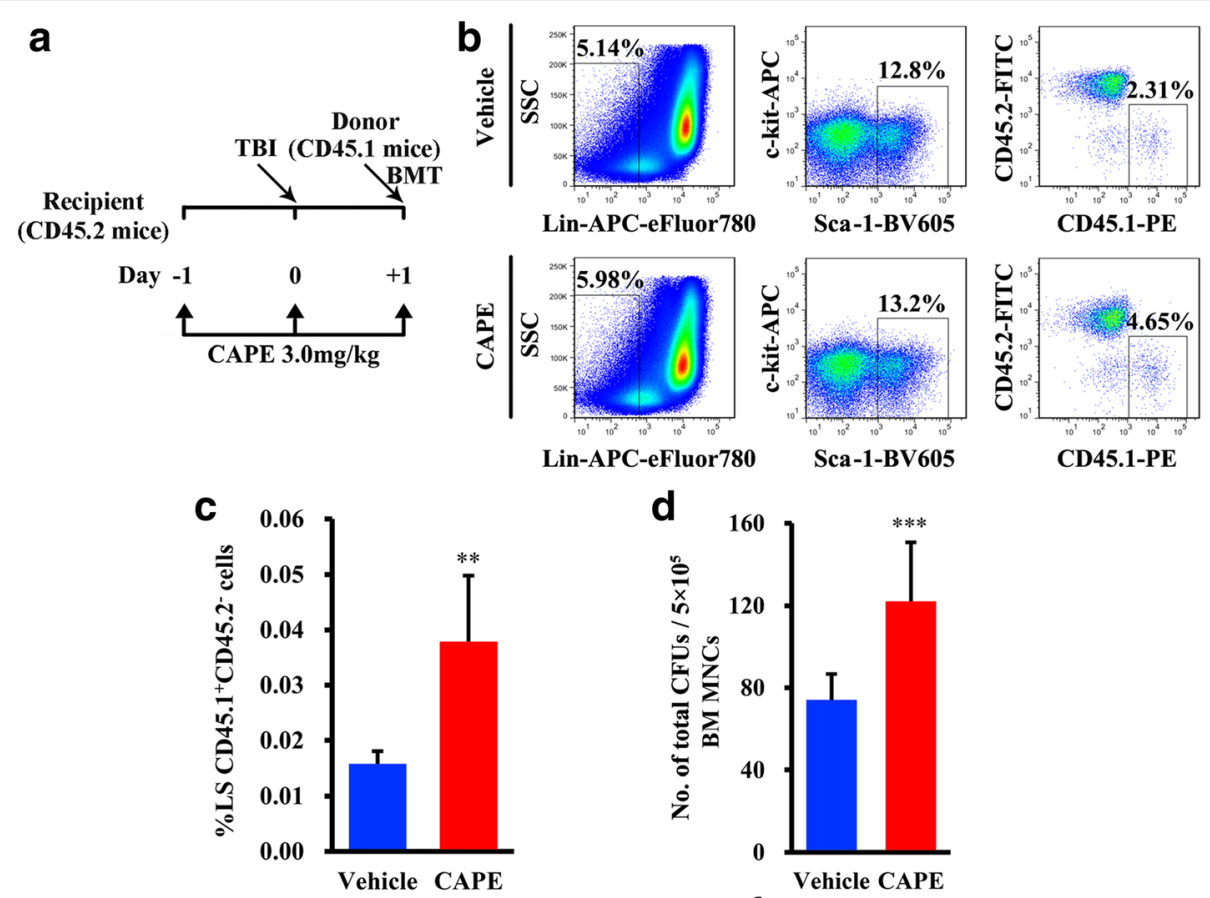

Lin-APC-eFluor780

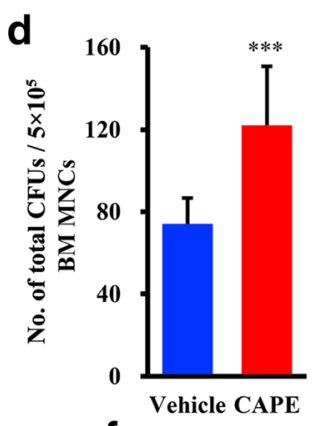

e
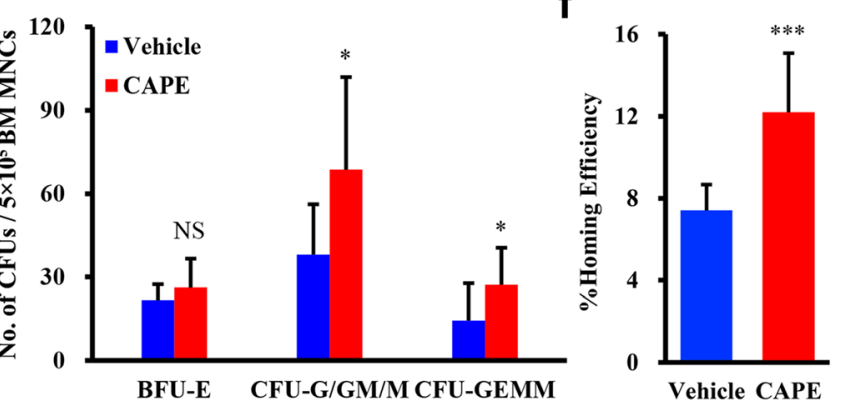

Fig. 2 CAPE promoted HSPC homing to the BM niche. a Schematic representation of the homing experiments. Lethally irradiated CD45.2 mice were transplanted with $2 \times 10^{7} \mathrm{CD} 45.1^{+} \mathrm{BM}$ MNCs and injected with $3.0 \mathrm{mg} / \mathrm{kg}$ CAPE or vehicle daily for 3 days. $\mathbf{b}$ Representative dot plots for murine BM Lin ${ }^{-} \mathrm{SCa}-1^{+} \mathrm{CD} 45.1^{+} \mathrm{CD} 45.2^{-}$cells. $\mathbf{c}$ Percentage of CD45. $1^{+} \mathrm{CD} 45.2^{-}$Lin ${ }^{-}$Sca- $1^{+}$cells in BM was increased by CAPE $(n=6)$. $\mathbf{d}$ Total CFU number generated from $5 \times 10^{5}$ BM MNCs. BM MNCs were harvested for CFU assays $20 \mathrm{~h}$ after BMT $(n=5)$. e Number of different types of CFU (BFU-E, CFU-G/GM/M and CFU-GEMM) generated from $5 \times 10^{5}$ BM MNCs. f Homing efficiency of CFUs calculated by comparing the homed CFU number with the initially injected CFU number. Blue indicates vehicle-treated group, red indicates CAPE-treated group; experiments repeated three times. Data presented as mean \pm SD. ${ }^{*} P<0.05$, ${ }^{*} P<0.01,{ }^{* *} P<0.001$, CAPE vs vehicle. TBI Total body irradiation, BM bone marrow, BMT bone marrow transplantation, CAPE caffeic acid phenethyl ester, SSC side scatter, CFU-G granulocyte colony-forming units, CFU-M macrophage colony-forming units, CFU-GEMM granulocyte-erythroblast-macrophage-megakaryocyte colony-forming units, CFU-GM granulocytemacrophage colony-forming units, MNC mononuclear cell, NS not significant

efficiency of HSPCs in the CAPE group significantly increased compared with the vehicle group (CAPE vs vehicle, $12.20 \pm 2.87 \%$ vs $7.41 \pm 1.27 \%, P=0.000057$; Fig. 2 f).

\section{CAPE enhanced short-term engraftment of HSPCs in mice} We showed that CAPE promoted HSPC homing to the BM. To further determine whether increased seeding of HSPCs to the BM as a result of CAPE treatment would result in better short-term engraftment of transplanted HSPCs than vehicle treatment, we analysed the CFU-S12 and BM CFU numbers on day 12 after transplantation. We found that more CFU-S12 grew on the spleen in the CAPE group than in the vehicle group (CAPE vs vehicle, $15.75 \pm 0.96$ vs $9 \pm 1.00$ CFU-S, $P=$ 0.000018; Fig. 3a, b). Similarly, an in-vitro CFU assay using $\mathrm{BM}$ cells harvested on day 12 after transplantation showed a significant increase in the total CFU number in the CAPE group $\left(238.7 \pm 23.12\right.$ CFUs per $1 \times 10^{5} \mathrm{BM}$ MNCs) compared with the vehicle group $(161.7 \pm 12.10$ CFUs per $1 \times 10^{5}$ BM MNCs, $P=0.0069$; Fig. $3 c$ ). Consistently, the BM cells from the CAPE treatment group formed a larger number of different types of CFUs than those from the vehicle group (CAPE vs vehicle: for BFU-E, $35.0 \pm 1.00$ vs $22.3 \pm 3.51, P=0.0039$; for CFU- 


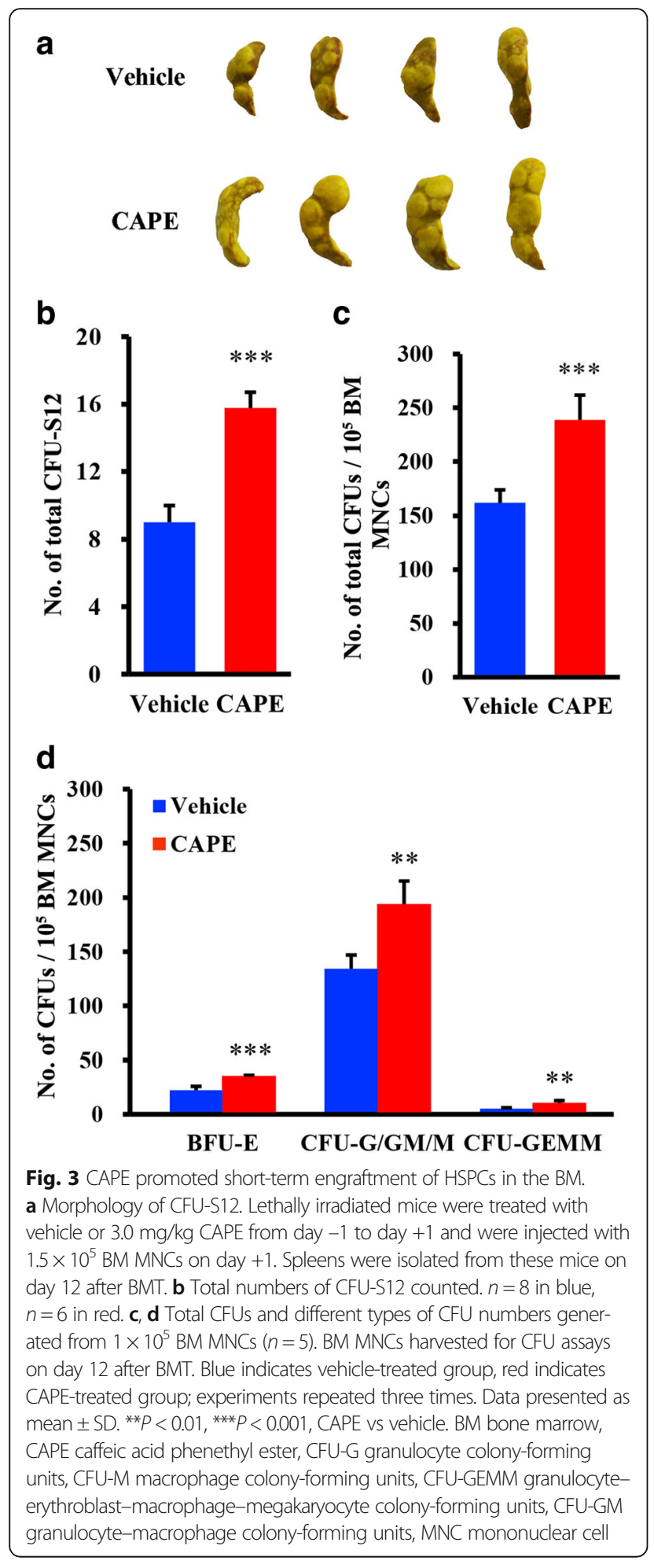

G/GM/M, $193.7 \pm 21.7$ vs $134.3 \pm 12.58, P=0.0149$; for CFU-GEMM, $10.0 \pm 2.65$ vs $5.0 \pm 1.00$ CFUs per $1 \times 10^{5}$ BM MNCs, $P=0.0069$; Fig. 3d). These results indicated that three doses of CAPE to the recipients significantly promoted short-term engraftment of HSPCs in mice.
CAPE promoted long-term engraftment of HSPCs in mice We then applied competitive repopulation models to evaluate the influence of CAPE on long-term engraftment of HSPCs. The $5 \times 10^{5}$ chimeric BM cells, obtained from CAPE-treated or vehicle-treated primary CD45.2 recipients $20 \mathrm{~h}$ following $\mathrm{CD} 45.1^{+} \mathrm{BM}$ cell transplantation, were transplanted into lethally irradiated secondary CD45.2 recipients. Then, $5 \times 10^{5} \mathrm{BM}$ cells from CD45.2 mice were co-transplanted into the lethally irradiated secondary CD45.2 recipients as competitor cells. Peripheral blood (PB) from secondary recipient mice was analysed for the presence of the primary donor (CD45.1) repopulation within 16 weeks after transplantation. The increase in the number of homed donor (CD45.1) haematopoietic cells in the mice that underwent CAPE administration was further revealed by increased engraftment of the primary donor $\mathrm{CD} 45.1^{+}$cells in the $\mathrm{PB}$ in the secondary recipients particularly at 8 , 12 and 16 weeks after transplantation (Fig. 4a). We analysed the lineage percentage of primary CD45.1 donor blood cells in the secondary recipient PB. Mice transplanted with $\mathrm{BM}$ cells from CAPE-treated primary recipients had more CD45.1-derived $\mathrm{B} 220^{+}, \mathrm{CD} 3 \mathrm{e}^{+}, \mathrm{CD} 11 \mathrm{~b}^{+}$, $\mathrm{Gr}-1^{+}$, and Ter- $119^{+}$cells than mice that received cells from vehicle-treated mice (Fig. 4b-f). Four months after transplantation, long-term donor CD45.1 ${ }^{+}$LSK cell engraftment rates in the $\mathrm{BM}$ increased by approximately 1.77 -fold in mice that received BM cells from the CAPEtreated primary recipient mice relative to mice that received cells from vehicle-treated primary recipients (Fig. 4g, h). These results strongly indicated that CAPE enhanced long-term engraftment of HSPCs, which was the result of CAPE-enhanced HSPC homing to the BM.

\section{CAPE upregulated the expression of HIF-1a, VEGF-A and SDF-1a}

To determine the mechanism by which CAPE promotes HSPC homing and engraftment, we harvested BM supernatants $20 \mathrm{~h}$ after transplantation from lethally irradiated mice treated with three doses of CAPE or vehicle. By applying a cytokine array, we found that the highest increases in VEGF-A occurred in the CAPE treatment group (Additional file 2: Figure S1). CAPE treatment also increased the levels of SDF-1 $\alpha$ in the BM (Additional file 3: Figure S1). We then used Q-PCR and western blot analysis to assess whether the expression of VEGF-A and SDF-1 $\alpha$ were activated in BM cells after CAPE treatment. Since HIF- $1 \alpha$ is a target gene of CAPE $[26,29]$, we also detected the expression of HIF- $1 \alpha$ in BMECs. The BMECs were incubated with CAPE at 0, 0.1 and $1.0 \mu \mathrm{g} / \mathrm{ml}$ for $24 \mathrm{~h}$. The results showed that 1.0 $\mu \mathrm{g} / \mathrm{ml}$ CAPE significantly upregulated the expression of HIF- $1 \alpha$, VEGF-A and SDF- $1 \alpha$ genes and proteins in BMECs (Fig. 5a, b). BM stromal cells (Methods for 

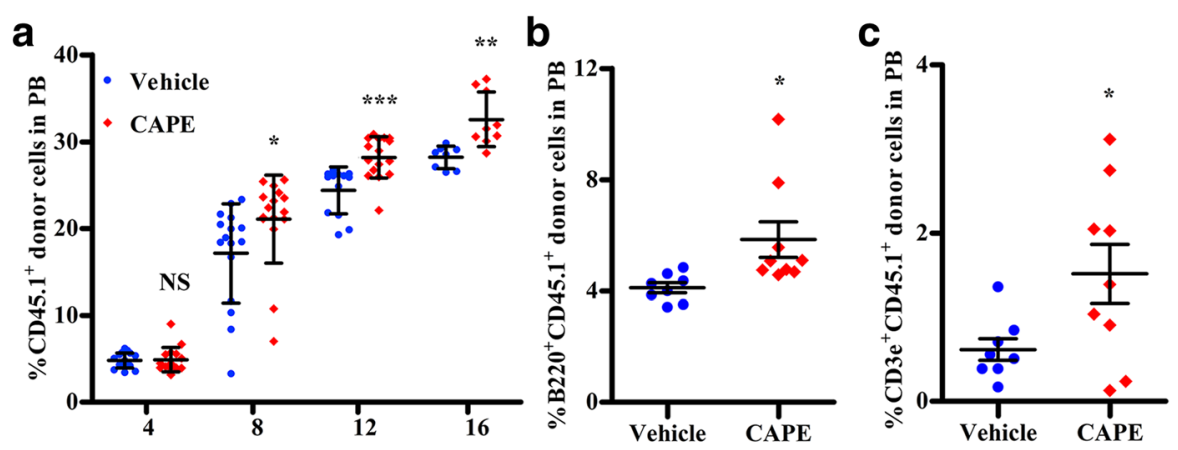

Time after transplantation (Weeks) 16 weeks after transplantation 16 weeks after transplantation

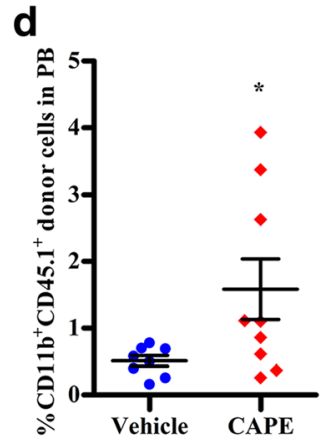

16 weeks after transplantation

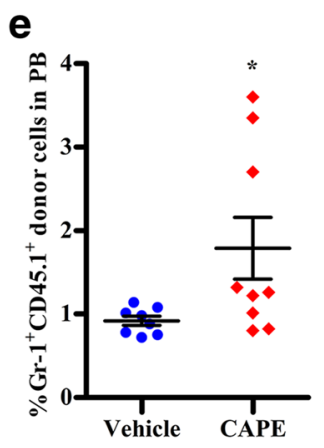

16 weeks after transplantation f

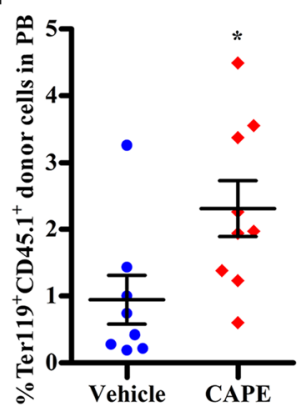

16 weeks after transplantation

g
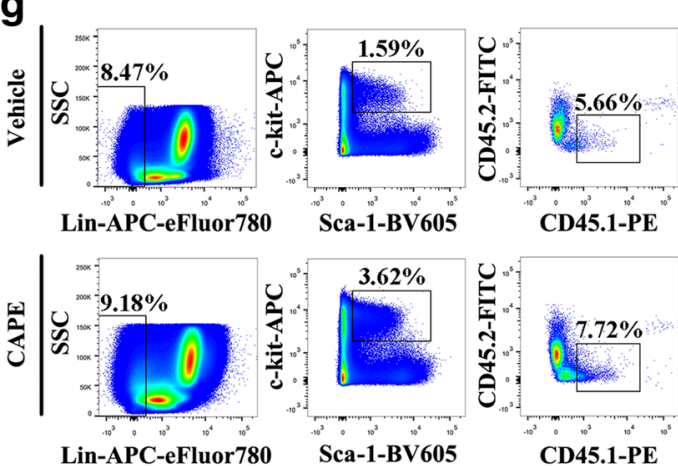

16 weeks after transplantation

h

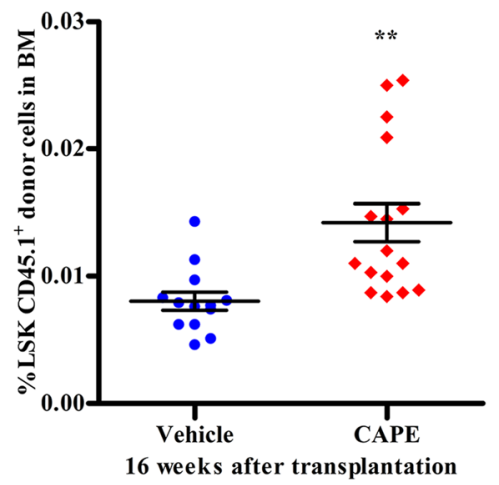

Fig. 4 CAPE promoted long-term engraftment of HSPCs in the BM. a Percentages of CD45.1 $1^{+}$cells in PB every 4 weeks after secondary transplantation until the 16 th week. BM MNCs $\left(5 \times 10^{5}\right)$ were harvested from the primary recipient mice $20 \mathrm{~h}$ after BMT and the various treatments and were then mixed with $5 \times 10^{5}$ competitor CD45.2 $2^{+}$BM MNCs and co-transplanted into irradiated CD45.2 mice (secondary recipients). Sixteen mice per group. b-f Donor CD45. $1^{+}$blood lineage cell percentages in the secondary recipient PB detected in the 16th week after secondary transplantation. $n=8$ in blue, $n=9$ in red. $\mathbf{g}$, $\mathbf{h}$ Representative dot plots and percentage of BM Lin ${ }^{-} \mathrm{Sca}-1^{+} \mathrm{C}-\mathrm{kit}{ }^{+} \mathrm{CD} 45.1^{+}$cells from secondary recipients $(n=16)$. BM cells were harvested from the secondary recipient mice at the 16 th week after competitive BMT. Blue indicates vehicle group, red indicates CAPE group. ${ }^{*} P<0.05$, ${ }^{* *} P<0.01,{ }^{* * *} P<0.001$, CAPE vs vehicle. BM bone marrow, CAPE caffeic acid phenethyl ester, SSC side scatter, NS not significant, PB peripheral blood

isolation and characterization of BM stromal cells are showed in Additional file 3) showed little alteration in the expression level of these genes (Additional file 4: Figure S2), suggesting that CAPE acts on BMECs and increases the expression of HIF- $1 \alpha$, VEGF-A and SDF- $1 \alpha$. We then performed an immunostaining experiment to detect the expression of these proteins in the BM niche of lethally irradiated mice $20 \mathrm{~h}$ after transplantation. CAPE treatment led to enhanced expression of HIF- $1 \alpha$ and SDF- $1 \alpha$ proteins in
BM cells and much stronger VEGF-A staining in BM stroma (Fig. 5c). These data suggested that CAPE-enhanced homing of HSPCs might be attributed to improvement of the BM niche by increasing SDF- $1 \alpha$ and VEGF-A levels.

\section{HIF-1a inhibitor PX-478 blocked CAPE-enhanced HSPC} homing

Several studies have suggested that VEGF and SDF- $1 \alpha$ are target genes of HIF- $1 \alpha[26,31,32]$. Based on these 


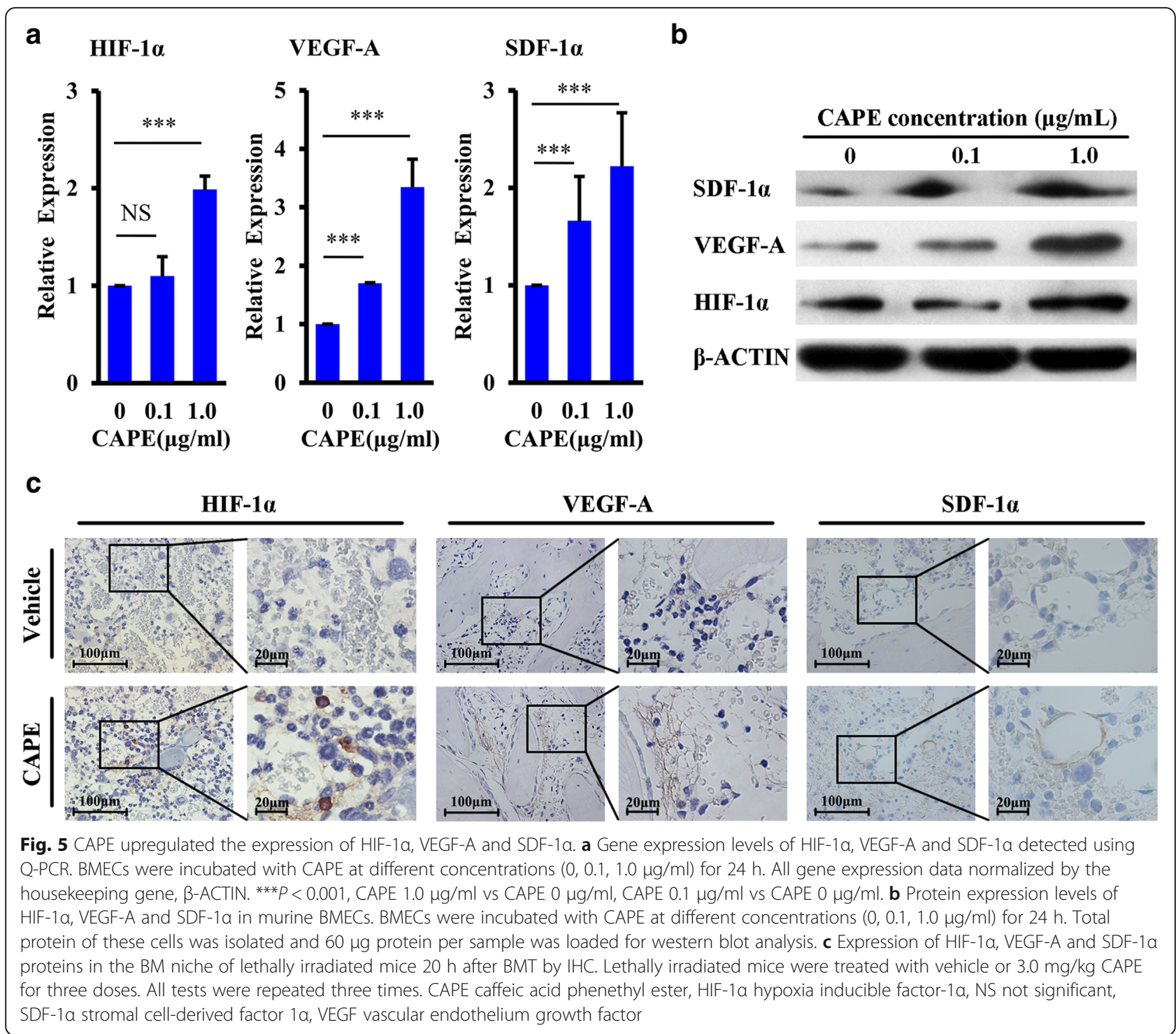

data, we hypothesized that HIF-1 $\alpha$, which can further regulate the expression of VEGF and SDF-1 $\alpha$, is the key target gene of CAPE. We then performed a homing experiment to assess whether blockade of HIF-1 $\alpha$ expression using an inhibitor attenuated the CAPE-enhanced HSPC homing effect. Lethally irradiated mice were injected intraperitoneally with vehicle or $3.0 \mathrm{mg} / \mathrm{kg}$ CAPE at the same schedule as shown in Fig. 2a, and they received BMT. For some mice, $5 \mathrm{mg} / \mathrm{kg}$ PX- 478 was administered just after daily CAPE injection. Twenty hours after transplantation, BM MNCs were harvested and cultured for CFU assays. The total number of CFUs in the CAPE group was greater than that in the vehicle group, and this difference was remarkably attenuated by injection with the HIF-1 $\alpha$ inhibitor PX-478 (vehicle vs CAPE vs CAPE and PX-478, 87.6 \pm 9.16 vs $141.7 \pm 7.61$ vs $76.1 \pm 8.34 \mathrm{CFU}$ number per $5 \times 10^{5} \mathrm{BM} \mathrm{MNCs}$ )
(Fig. 6a). The CAPE-mediated increase in HSPC homing efficiency was significantly inhibited by PX-478 (Fig. 6b). These results indicated that CAPE-enhanced HSPC homing to the $\mathrm{BM}$ was primarily dependent on regulation of HIF- $1 \alpha$.

\section{Discussion}

HSCT is an effective and life-saving therapeutic strategy for patients with malignant and non-malignant disorders. Infused HSPCs are required to undergo several crucial processes to complete repopulation, including efficient homing to the BM from the circulating blood, engraftment in the BM and regeneration of all blood cell types. There are at least two ways to enhance homing of infused HSPCs to the BM, especially for limited numbers of HSPCs from CB. In-vitro manipulation of HSPCs to increase their number is an ex-vivo approach to increase HSPC homing and engraftment to the BM [33]. 

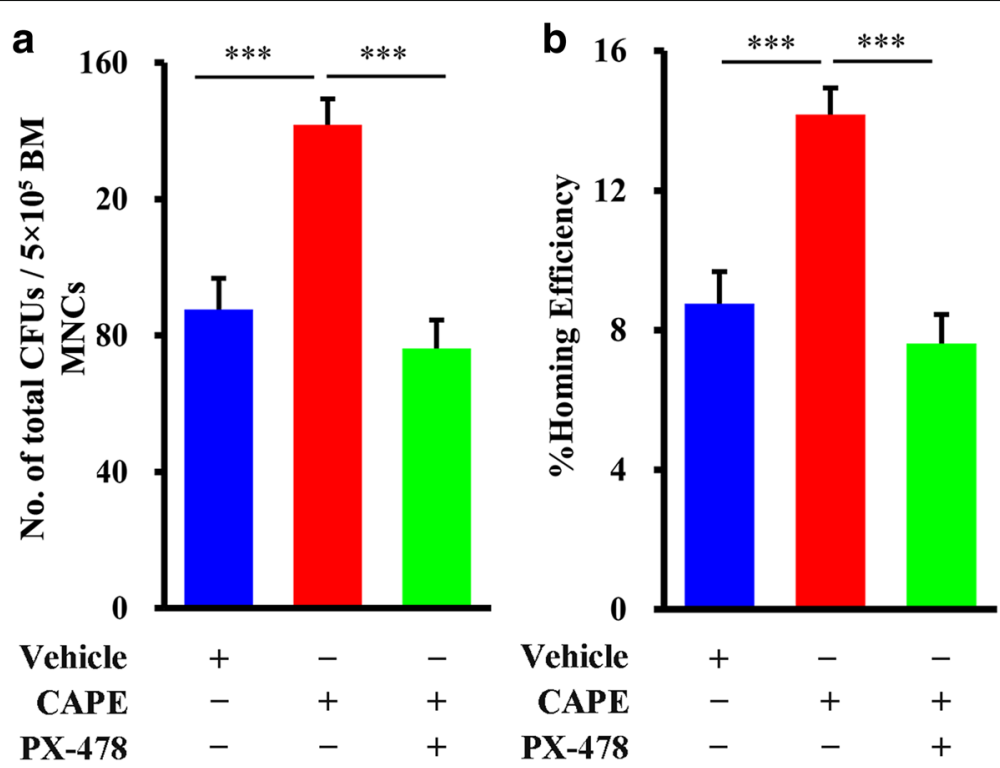

Fig. 6 HIF-1a inhibitor PX-478 blocked CAPE-enhanced HSPC homing. a Total CFU number generated from $5 \times 10^{5}$ BM MNCS $(n=5)$. Lethally irradiated mice were injected intraperitoneally with vehicle or $3.0 \mathrm{mg} / \mathrm{kg}$ CAPE from day -1 to day +1 , and received BMT on day +1 . For some mice, 5 mg/kg PX-478 was administered just after CAPE injection. Twenty hours after BMT, BM MNCs were harvested and cultured for CFU assays. Experiment repeated three times. $\mathbf{b}$ Homing efficiency calculated by comparing the homed CFU numbers with the initially injected CFU numbers. Data presented as mean \pm SD. ${ }^{* *} P<0.001$, CAPE vs Vehicle, CAPE vs CAPE + PX-478. BM bone marrow, CAPE caffeic acid phenethyl ester, CFU colony-forming unit, MNC mononuclear cell

Improving chemotactic or adherent ability of HSPCs to the BM by ex-vivo treatment of HSPCs is also proved to be an effective strategy to enhance HSPC seeding to the BM. Some efforts include short-term exposure of HSPCs to PGE2, and short-term treatment of HSPCs with hyperthermia or hyperbaric oxygen $[10,34,35]$. To enhance HSPC homing and engraftment in the BM, another strategy is to give drugs to patients receiving HSPC transplantation [36], which is a relatively simple method that avoids the problems caused by ex-vivo manipulation. The drug sitagliptin has been reported to be used in patients for improving homing and engraftment of CB stem cells in clinical trials, which functions in vivo mainly via regulating the chemotactic activity of the transfused HSPCs [37]. Given that several chemotactic factors in the BM microenvironment have been proved to be involved in the retention of HSPCs, using drugs to improve the $\mathrm{BM}$ niche of patients is becoming a novel strategy [38, 39]. However, development of this kind of drug is still a challenge. Here, we found that CAPE, a natural compound extracted from honeybee hives, showed the potential to become this kind of candidate drug mainly via regulating the $\mathrm{BM}$ microenvironment.

CAPE is found in many plants and can also be synthesized by reacting caffeic acid with phenethyl alcohols [40, 41]. The various effects of CAPE are related to the dose, target cell type and disease model. In our study, we found that treatment of the recipients with CAPE enhanced HSPC homing and engraftment in the BM. By applying survival rate experiments in lethally irradiated mice with limited BM cell transplantation and CAPE treatment, we confirmed that CAPE injection to lethally irradiated recipients had a notably positive role in improving the survival rate and haematopoietic repopulation in mice receiving BMT. The dose and frequency of CAPE injection were different from that used in other disease models. For HSPC homing and engraftment experiments, a frequently used mouse model-that is, lethally irradiation with BMT $[10,30]$-was chosen to evaluate the effect of CAPE. An optimal schedule for administration of CAPE at $3.0 \mathrm{mg} / \mathrm{kg}$ to the recipients from day -1 to +1 was further confirmed to be effective in significantly improving HSPC homing and subsequent short-term and long-term engraftment.

Increasing evidence has indicated that different mechanisms are involved in the various functions of CAPE, including induction of HO-1 expression, activation of the ERK1/2-CREB signalling cascade and inhibition of NF- $\mathrm{KB}$ signals in different cell contexts and different disease models [42-45]. We found that CAPE upregulated the HIF- $1 \alpha$ and SDF- $1 \alpha$ gene and protein expression in BMECs, which further supports the hypothesis that CAPE has the ability to improve haematopoietic cell homing by regulating the BM niche (Fig. 7). SDF- $1 \alpha$ is primarily expressed and secreted by BM niche cells, such as endothelial cells, stromal cells and osteoblasts. The SDF- $1 \alpha$ level in the BM niche is a critical determinant for efficient HSPC recruitment and homing [4, 10, 46]. 


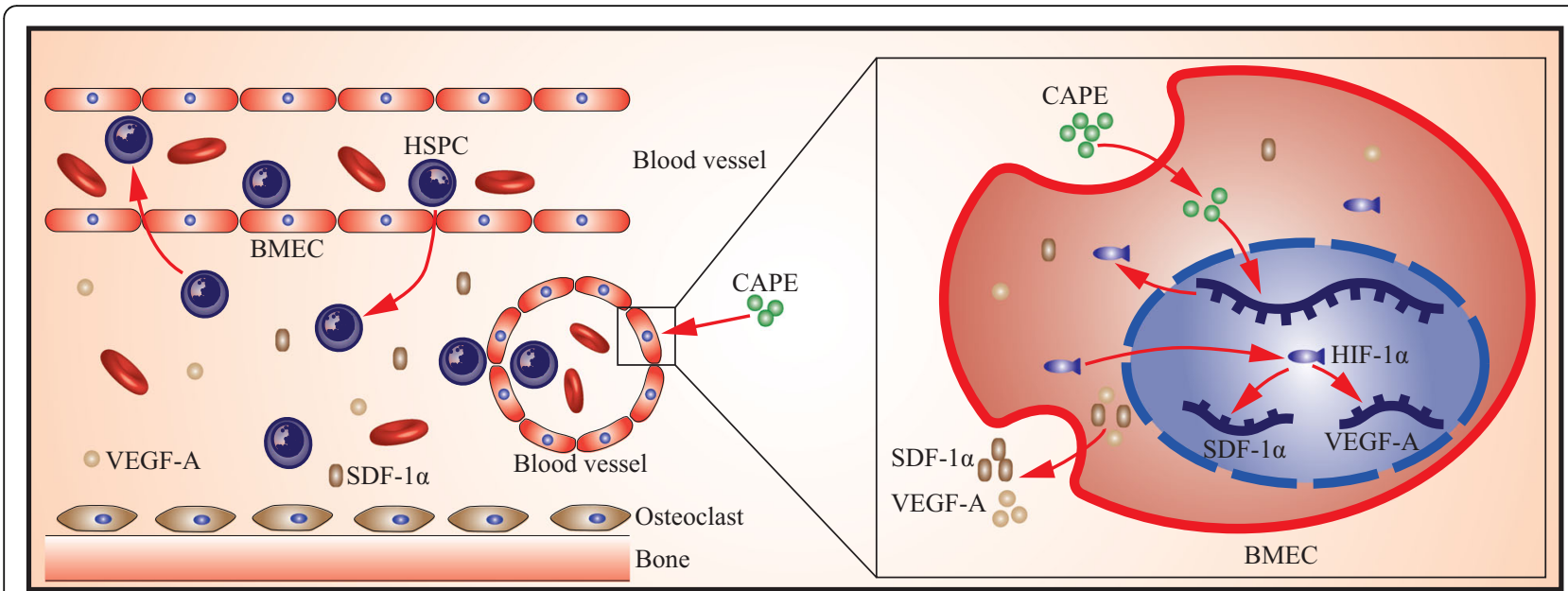

Fig. 7 Graphic representation of the regulation mechanism of CAPE in the improvement of HSPC homing. Left: process of HSPC homing and engraftment in the BM, including HSPC rolling in vessels, migrating across BMECs and lodging in the BM niche. Right: CAPE induces expression of HIF-1 a protein, activating downstream SDF-1a and VEGF-A gene expression in BMECs. Thus, CAPE creates a better chemotactic and survival environment for transplanted HSPCs and promotes HSPC homing and engraftment. Picture drawn by Dr Hailiang Li. BMEC bone marrow endothelial cell, CAPE caffeic acid phenethyl ester, HIF-1a hypoxia inducible factor-1a, HSPC haematopoietic stem/progenitor cell, SDF-1a stromal cell-derived factor 1a, VEGF vascular endothelium growth factor

CAPE-enhanced SDF- $1 \alpha$ immunostaining in BM microvessels suggested that the target cells of CAPE in irradiated BM were BMECs. BM mesenchymal-like stromal cells were not the target cells of CAPE, as evidenced by their non-responsiveness to CAPE. In addition to SDF$1 \alpha$, VEGF-A, which functions as a survival factor for endothelial cells and haematopoietic stem cells, was also increased in the BM niche. Taken together, the increased SDF- $1 \alpha$ and VEGF-A concentration in the BM niche created a better chemotactic and survival environment for transplanted HSPCs and led to increased HSPC homing to the damaged BM. Several studies have indicated that both SDF- $1 \alpha$ and VEGF are downstream target genes of the transcriptional factor HIF-1 $\alpha$ [31, 32]. In our experiments, we found that CAPE upregulated the expression of HIF-1 $\alpha$. By performing a HIF- $1 \alpha$ inhibitor blocking experiment, we further confirmed that HIF- $1 \alpha$ was a key point for inhibiting CAPE-induced HSPC homing. In future, more work needs to be done to clarify the mechanism of CAPE in activating HIF- $1 \alpha$ transcription and extend these findings. Furthermore, comparison of the effect of CAPE derivatives with that of CAPE might be helpful to find more efficient candidate drugs for improvement of HSPC homing and engraftment in the BM.

\section{Conclusions}

Our data are the first to show that CAPE administration to irradiated BMT recipients promotes recruitment and homing of HSPCs to the BM niche and subsequently improves the short-term and long-term engraftment of HSPCs in a BM transplantation model.
The mechanism appears to involve activation of HIF- $1 \alpha$ and downstream SDF- $1 \alpha$ and VEGF-A gene expression in BMECs and creating a better BM niche for HSPC homing. Thus, we present a potent application for CAPE in improving homing and engraftment outcomes in patients receiving $\mathrm{HSCT}$.

\section{Additional files}

Additional file 1: Table S1. Presenting primers for Q-PCR. (DOCX $11 \mathrm{~kb}$ )

Additional file 2: Figure S1. Showing proteins detected in BM supernatants from lethally irradiated mice treated with three doses of CAPE or vehicle $20 \mathrm{~h}$ after transplantation, $n=3$. Femurs of three mice per group were flushed using $5 \mathrm{ml}$ PBS. Pooled supernatants per group were collected after centrifugation, freeze-dried and resuspended in 120 $\mu \mathrm{PBS}$. Cytokine array performed using the RayBio ${ }^{\oplus}$ Mouse Cytokine Antibody Array G-Series 3 (RayBiotech) following the manufacturer's instructions. (TIF $1214 \mathrm{~kb}$ )

Additional file 3: Supplementary methods for isolation and characterization of BM stromal cells. (DOCX $10 \mathrm{~kb}$ )

Additional file 4: Figure S2. Showing expression of HIF-1a, VEGF-A and SDF-1a in primary mouse BMSCs. $a, b$ Gene and protein expression levels of HIF-1a, VEGF-A and SDF-1a in primary mouse BMSCs. BMSCs were incubated with CAPE at different concentrations $(0,0.1,1.0 \mu \mathrm{g} / \mathrm{ml})$ respectively for $24 \mathrm{~h}$. All gene expression data normalized by the housekeeping gene, $\beta$-ACTIN. Every test was repeated three times. (TIF $323 \mathrm{~kb}$ )

\section{Abbreviations}

BFU-E: Erythrocyte burst-forming unit; BM: Bone marrow; BMEC: Bone marrow endothelial cell; BMSC: Bone marrow stromal cell; BMT: Bone marrow transplantation; CAPE: Caffeic acid phenethyl ester; CB: Cord blood; CFU: Colony-forming unit; CFU-G: Granulocyte colony-forming units; CFU-GEMM: Granulocyte-erythroblast-macrophage-megakaryocyte colony-forming units; CFU-GM: Granulocyte-macrophage colony-forming units; CFU-Meg: Megakaryocyte colony-forming units; DMSO: Dimethyl sulphoxide; FVS510: Fixable Viability Stain 510; HIF-1a: Hypoxia inducible factor-1a; HO-1: Haeme oxygenase-1; HSCT: Haematopoietic stem cell 
transplantation; HSPC: Haematopoietic stem/progenitor cell; Lin: Lineage; LSK: Lin ${ }^{-}$Sca- $1^{+}{ }^{+}-\mathrm{Kit}^{+}$; MNC: Mononuclear cell; PB: Peripheral blood; PGE2: Prostaglandin E2; SCF: Stem cell factor; SDF-1a: Stromal cell-derived factor 1a; SSC: Side scatter; TBI: Total body irradiation; VEGF: Vascular endothelium growth factor

\section{Acknowledgements}

The authors thank Dr Hailiang Li, ZhuHai People's Hospital, Zhuhai, China, for designing and drawing Fig. 7.

\section{Funding}

This work was supported by National High Technology Research and Development Program of China (No. 2013AA020107), National Key R\&D Program of China (No. 2017YFA0103100), Nature Science Foundation of China (No. 81472908, 31401260) and Guangzhou Health Care and Cooperative Innovation Major Project (No. 201400000003-4).

\section{Availability of data and materials}

All data generated or analysed during this study are included in the published article and its supplementary information files.

\section{Authors' contributions}

$X C$ performed all the experiments, and participated in the research design, data analysis, and manuscript writing. YH assisted in the animal experiments. BZ participated in research design and data analysis. YiL participated in the transplantation experiments. SW assisted in the transplantation experiments. $\mathrm{TL}$ assisted in the animal experiments. ZD assisted in the animal experiments. ZF participated in immunophenotype analysis. JZ, LH and WY assisted in designing the research. YanL designed the study, analysed the data, and wrote the manuscript. XP designed the research, analysed the data, and revised the manuscript. All authors read and approved the final manuscript.

\section{Ethics approval}

All animal experiments performed in accordance with the institutional guidelines for animal research, and approved by the Animal Center Committee of the Academy of Military Medical Sciences, Beijing, China (Permit Number: AMMS-13-2015-009).

\section{Consent for publication}

All authors read and approved the manuscript for publication.

\section{Competing interests}

The authors declare that they have no competing interests.

\section{Publisher's Note}

Springer Nature remains neutral with regard to jurisdictional claims in published maps and institutional affiliations.

\section{Author details \\ ${ }^{1}$ School of Laboratory Medicine and Biotechnology, Southern Medical University, No. 1838 Guangzhou Avenue North, Baiyun District, Guangzhou 510515, China. ${ }^{2}$ Stem Cell and Regenerative Medicine Lab, Beijing Institute of Transfusion Medicine, No. 27 Taiping Road, Haidian District, Beijing 100850, China. ${ }^{3}$ South China Institute of Biomedicine, No. 1 Luoxuan 4th Road, Haizhu District, Guangzhou 510005, China. ${ }^{4}$ Guangdong Medical University, No. 1 Xincheng Road, Dongguan 523808, China.}

Received: 25 April 2017 Revised: 24 August 2017 Accepted: 23 October 2017 Published online: 07 November 2017

\section{References}

1. Thomas ED. A history of allogeneic hematopoietic cell transplantation. Oxford: Wiley-Blackwell; 2009. p. 1-7.

2. Armitage JO. The history of autologous hematopoietic cell transplantation. In: Thomas' hematopoietic cell transplantation. Hoboken, NJ: WileyBlackwell; 2009. p. 8-14.

3. Christopherson 2nd KW, Cooper S, Broxmeyer HE. Cell surface peptidase CD26/DPPIV mediates G-CSF mobilization of mouse progenitor cells. Blood. 2003;101:4680-6.
4. Kim $\mathrm{CH}$, Broxmeyer HE. In vitro behavior of hematopoietic progenitor cells under the influence of chemoattractants: stromal cell-derived factor-1, steel factor, and the bone marrow environment. Blood. 1998;91:100-10.

5. Levesque JP, Hendy J, Takamatsu Y, Simmons PJ, Bendall L. Disruption of the CXCR4/CXCL12 chemotactic interaction during hematopoietic stem cell mobilization induced by GCSF or cyclophosphamide. J Clin Invest. 2003;111: 187-96.

6. Liles WC, Broxmeyer HE, Rodger E, et al. Mobilization of hematopoietic progenitor cells in healthy volunteers by AMD3100, a CXCR4 antagonist. Blood. 2003;102:2728-30.

7. Peled A, Petit I, Kollet O, et al. Dependence of human stem cell engraftment and repopulation of NOD/SCID mice on CXCR4. Science. 1999; 283:845-8.

8. Copelan EA. Hematopoietic stem-cell transplantation. N Engl J Med. 2006; 354:1813-26.

9. Cutler C, Multani P, Robbins D, et al. Prostaglandin-modulated umbilical cord blood hematopoietic stem cell transplantation. Blood. 2013;122:3074-81.

10. Hoggatt J, Singh P, Sampath J, Pelus LM. Prostaglandin E2 enhances hematopoietic stem cell homing, survival, and proliferation. Blood. 2009;113: 5444-55.

11. Porter RL, Georger MA, Bromberg $\mathrm{O}$, et al. Prostaglandin E2 increases hematopoietic stem cell survival and accelerates hematopoietic recovery after radiation injury. Stem Cells. 2013;31:372-83.

12. Sackstein R. Re: "Ex vivo fucosylation improves human cord blood engraftment in NOD-SCID IL-2Rgamma(null) mice". Exp Hematol. 2012;40: 518-9. author reply 519-20.

13. Levesque JP, Helwani FM, Winkler IG. The endosteal 'osteoblastic' niche and its role in hematopoietic stem cell homing and mobilization. Leukemia. 2010;24:1979-92.

14. Ratajczak MZ. A novel view of the adult bone marrow stem cell hierarchy and stem cell trafficking. Leukemia. 2015;29:776-82.

15. Ara T, Tokoyoda K, Sugiyama T, Egawa T, Kawabata K, Nagasawa T. Longterm hematopoietic stem cells require stromal cell-derived factor-1 for colonizing bone marrow during ontogeny. Immunity. 2003;19:257-67.

16. Seitz G, Boehmler AM, Kanz L, Mohle R. The role of sphingosine 1phosphate receptors in the trafficking of hematopoietic progenitor cells. Ann N Y Acad Sci. 2005;1044:84-9.

17. Adamiak M, Borkowska S, Wysoczynski M, et al. Evidence for the involvement of sphingosine-1-phosphate in the homing and engraftment of hematopoietic stem cells to bone marrow. Oncotarget. 2015;6:18819-28.

18. Golan K, Vagima Y, Ludin A, et al. S1P promotes murine progenitor cell egress and mobilization via S1P1-mediated ROS signaling and SDF-1 release. Blood. 2012;119:2478-88.

19. Kimura T, Boehmler AM, Seitz G, et al. The sphingosine 1-phosphate receptor agonist FTY720 supports CXCR4-dependent migration and bone marrow homing of human CD34+ progenitor cells. Blood. 2004;103:4478-86.

20. Kishimoto N, Kakino Y, Iwai K, Mochida K, Fujita T. In vitro antibacterial, antimutagenic and anti-influenza virus activity of caffeic acid phenethyl esters. Biocontrol Sci. 2005;10:155-61.

21. Velazquez C, Navarro M, Acosta A, et al. Antibacterial and free-radical scavenging activities of Sonoran propolis. J Appl Microbiol. 2007;103:1747-56.

22. Ojeda-Contreras AJ, Hernández-Martínez J, Domínguez Z, et al. Utilization of caffeic acid phenethyl ester to control alternaria alternata rot in tomato (lycopersicon esculentum mill.) fruit. J Phytopathol. 2008;156:164-73.

23. Lee HS, Lee SY, Park SH, et al. Antimicrobial medical sutures with caffeic acid phenethyl ester and their in vitro/in vivo biological assessment. Med Chem Commun. 2012;4:777-82.

24. Jo SY, Lee N, Hong SM, Jung HH, Chae SW. Caffeic acid phenethyl ester inhibits diesel exhaust particle-induced inflammation of human middle ear epithelial cells via NOX4 inhibition. Ann Otol Rhinol Laryngol. 2013;122:595-600.

25. Cunha FMD, Duma D, Assreuy J, et al. Caffeic acid derivatives: in vitro and in vivo anti-inflammatory properties. Free Radic Res. 2004;38:1241-53.

26. Choi D, Han J, Lee Y, et al. Caffeic acid phenethyl ester is a potent inhibitor of HIF prolyl hydroxylase: structural analysis and pharmacological implication. J Nutr Biochem. 2010;21:809.

27. Kallio PJ, Wilson WJ, O'Brien S, Makino Y, Poellinger L. Regulation of the hypoxia-inducible transcription factor 1alpha by the ubiquitin-proteasome pathway. J Biol Chem. 1999:274:6519.

28. Tian YM, Mole DR, Ratcliffe PJ, Gleadle JM. Characterization of different isoforms of the HIF prolyl hydroxylase PHD1 generated by alternative initiation. Biochem J. 2006;397:179-86. 
29. Liu Y, Zhang B, Zhang J, et al. CAPE promotes the expansion of human umbilical cord blood-derived hematopoietic stem and progenitor cells in vitro. Sci China Life Sci. 2014;57:188-94.

30. Khurana S, Buckley S, Schouteden S, et al. A novel role of BMP4 in adult hematopoietic stem and progenitor cell homing via Smad independent regulation of integrin-alpha4 expression. Blood. 2013;121:781-90.

31. Chen Y, Fu L, Han Y, et al. Testosterone replacement therapy promotes angiogenesis after acute myocardial infarction by enhancing expression of cytokines HIF-1a, SDF-1a and VEGF. Eur J Pharmacol. 2012;684:116-24.

32. Youn SW, Lee SW, Lee J, et al. COMP-Ang1 stimulates HIF-1alpha-mediated SDF-1 overexpression and recovers ischemic injury through BM-derived progenitor cell recruitment. Blood. 2011;117:4376-86.

33. Li X, Ma X, Luo Y, Danyue P, Zhou D, Liu L. Inhibition of both activated p38 MAPK and mTOR C1 potentiates the effect of SR1 on promotion of hematopoietic stem cell expansion ex vivo. Blood. 2015:126:381.

34. Wierenga PK, Konings AW. Studies on the hyperthermic sensitivity of the murine hematopoietic stem cell compartment. I. Heat effects on clonogenic stem cells and progenitors. Exp Hematol. 1993;21:608-13.

35. Aljitawi OS, Xiao Y, Eskew JD, et al. Hyperbaric oxygen improves engraftment of ex-vivo expanded and gene transduced human CD34 + cells in a murine model of umbilical cord blood transplantation. Blood Cells Mol Dis. 2014:52:59.

36. Olson TS, Caselli A, Otsuru S, et al. Megakaryocytes promote murine osteoblastic HSC niche expansion and stem cell engraftment after radioablative conditioning. Blood. 2013;121:5238-49.

37. Farag SS, Srivastava S, Messina-Graham S, et al. In vivo DPP-4 inhibition to enhance engraftment of single-unit cord blood transplants in adults with hematological malignancies. Stem Cells Dev. 2013;22:1007.

38. Rossi L, Manfredini R, Bertolini F, et al. The extracellular nucleotide UTP is a potent inducer of hematopoietic stem cell migration. Blood. 2007;109:533.

39. Ratajczak MZ, Kim CH, Abdel-Latif A, et al. A novel perspective on stem cell homing and mobilization: review on bioactive lipids as potent chemoattractants and cationic peptides as underappreciated modulators of responsiveness to SDF-1 gradients. Leukemia. 2012;26:63.

40. Chen HC, Chen JH, Chang C, Shieh CJ. Optimization of ultrasoundaccelerated synthesis of enzymatic caffeic acid phenethyl ester by response surface methodology. Ultrason Sonochem. 2011;18:455-9.

41. Chen HC, Ju HY, Twu YK, et al. Optimized enzymatic synthesis of caffeic acid phenethyl ester by RSM. N Biotechnol. 2010;27:89-93.

42. Scapagnini G, Foresti R, Calabrese V, Giuffrida Stella AM, Green CJ, Motterlini R. Caffeic acid phenethyl ester and curcumin: a novel class of heme oxygenase-1 inducers. Mol Pharmacol. 2002;61:554.

43. Toratani A. Caffeic acid phenethyl ester ameliorates depression- and anxiety-like behaviors of mice exposed to chronic mild stress. J Neurophysiol Neurol Disord. 2014;1:202.

44. Natarajan K, Singh S, Burke TR, Grunberger D, Aggarwal BB. Caffeic acid phenethyl ester is a potent and specific inhibitor of activation of nuclear transcription factor NF-kappa B. Proc Natl Acad Sci U S A. 1996:93:9090-5.

45. Choi EY, Choe SH, Hyeon JY, Choi JI, Choi IS, Kim SJ. Effect of caffeic acid phenethyl ester on prevotella intermedia lipopolysaccharide-induced production of proinflammatory mediators in murine macrophages. J Periodontal Res. 2015;50:737-47.

46. Lapidot T, Dar A, Kollet O. How do stem cells find their way home? Blood. 2005;106:1901-10.

\section{Submit your next manuscript to BioMed Central and we will help you at every step:}

- We accept pre-submission inquiries

- Our selector tool helps you to find the most relevant journal

- We provide round the clock customer support

- Convenient online submission

- Thorough peer review

- Inclusion in PubMed and all major indexing services

- Maximum visibility for your research

Submit your manuscript at www.biomedcentral.com/submit
C Biomed Central 